ARTICLE

https://doi.org/10.1038/s41467-020-19271-1

\title{
Engineered fluoride sensitivity enables biocontainment and selection of genetically-modified yeasts
}

\author{
Justin I. Yoo ${ }^{1}$, Susanna Seppälä (i) ${ }^{1} \&$ Michelle A. O’Malley (iD ${ }^{1 凶}$
}

Biocontainment systems are needed to neutralize genetically modified organisms (GMOs) that pose ecological threats outside of controlled environments. In contrast, benign selection markers complement GMOs with reduced fitness. Benign selection agents serve as alternatives to antibiotics, which are costly and risk spread of antibiotic resistance. Here, we present a yeast biocontainment strategy leveraging engineered fluoride sensitivity and DNA vectors enabling use of fluoride as a selection agent. The biocontainment system addresses the scarcity of platforms available for yeast despite their prevalent use in industry and academia. In the absence of fluoride, the biocontainment strain exhibits phenotypes nearly identical to those of the wildtype strain. Low fluoride concentrations severely inhibit biocontainment strain growth, which is restored upon introduction of fluoride-based vectors. The biocontainment strategy is stringent, easily implemented, and applicable to several eukaryotes. Further, the DNA vectors enable genetic engineering at reduced costs and eliminate risks of propagating antibiotic resistance.

\footnotetext{
${ }^{1}$ Department of Chemical Engineering, University of California Santa Barbara, Santa Barbara, CA 93106, USA. 凶email: momalley@ucsb.edu
} 
$\mathrm{R}$ apid advancements in synthetic biology augment both our ability to engineer cellular functions as well as concerns associated with genetically modified organisms (GMOs). GMOs have been engineered to produce biofuels, chemicals, and pharmaceuticals at industrial scale ${ }^{1-4}$, and the design and construction of microbial genomes ${ }^{5-9}$ promise even greater capacity to engineer cells with precisely defined functions. However, these advances amplify concerns surrounding health and ecological risks posed by GMOs that house hazardous genetic material or have a fitness advantage over microbes found in natural ecosystems ${ }^{10-12}$. The potential release of GMOs is particularly concerning due to the emergence of do-it-yourself synthetic biology kits enabling construction of GMOs without the physical containment strategies present in academia and industry. In addition to biocontainment, most biotechnological applications would derive great benefit from benign selection markers as alternatives to antibiotics, which are costly and may incur risk of propagating antibiotic resistance through overuse of antibiotics and horizontal gene transfer (HGT) even from lysed cells ${ }^{13-15}$.

While methods for biocontainment of bacteria have advanced rapidly ${ }^{11,12}$, only two strategies have been demonstrated in the yeast Saccharomyces cerevisiae ${ }^{16,17}$ despite their extensive use as production platforms in academia ${ }^{18,19}$ and in industry ${ }^{20,21}$. Moreover, the two biocontainment strategies presented for $S$. cerevisiae require exogenous ligands and cellular machinery for survival and depend on inducible transcription of essential genes. This design strategy renders the safeguard mechanisms susceptible to inactivating mutations, which were indeed observed ${ }^{16,17}$. In contrast, a permissive state independent of mutable systems would markedly reduce the likelihood of biocontainment inactivation. Further, the ideal eukaryotic biocontainment strategy is compatible with various microorganisms. The generality of such a biocontainment system is increasingly important as non-model eukaryotic organisms continue to be developed as production platforms.

The mechanism underlying eukaryotic fluoride tolerance was recently elucidated in three eukaryotes and depends on the presence of at least one fluoride exporter protein, FEX1 or FEX2 22 . Recognizing the broad utility of this stringent selection mechanism, we sought to extend the application of fluoride sensitivity to two pressing needs in synthetic biology: biocontainment and alternative selection markers. Accordingly, we present a yeast biocontainment strain that is highly sensitive to fluoride and a complementary set of DNA vectors reliant on fluoride-based selection (Fig. 1).

\section{Results}

Biocontainment strain construction. To sensitize yeast to fluoride, we previously generated a $S$. cerevisiae strain lacking both native fluoride exporter $(F E X 1 / 2)$ genes (Fig. 2a $)^{23}$. Accordingly, the knockout strain (i.e., the biocontainment strain) is highly sensitive to fluoride exhibiting an $\mathrm{IC}_{50}$ of $46.6 \mu \mathrm{M} \mathrm{NaF}$, which is approximately three orders of magnitude lower than that of the wildtype (WT) parent strain $(21.9 \mathrm{mM} \mathrm{NaF})$ and agrees with the initial report ${ }^{22}$ (Fig. 2b). Strikingly, growth of the biocontainment strain is severely inhibited by $210.5 \mu \mathrm{M} \mathrm{NaF}$ (Fig. 2a, b), which is equal to the U.S. EPA standard for drinking water quality ${ }^{24}$ and significantly lower than concentrations observed in groundwater, where fluoride concentrations vary significantly depending on location and associated environmental factors ${ }^{25}$. While a review of $>38,000$ U.S. wells indicates that a majority $(>80 \%)$ contain $\left[\mathrm{F}^{-}\right]<36.8 \mu \mathrm{M}$, many of these sites are proximal to those with high fluoride concentrations. Therefore, we speculate that intermixing of groundwater in regions with high $\left[\mathrm{F}^{-}\right]$ may increase the likelihood of growth inhibition of a biocontainment strain released into nature. In addition to spatial variation, temporal fluctuation of fluoride concentration should be considered. As rainwater is poor in fluoride, surface water (e.g., lakes, rivers) and shallow groundwater often contain lower fluoride concentrations due to dilution by rain ${ }^{26}$. Similarly, areas with high rainfall can be expected to contain lower levels of fluoride. Thus, while fluoride sensitivity may serve as a robust biocontainment measure in areas known to have high fluoride concentrations, care should be taken to evaluate local fluoride abundance. Accordingly, fluoride-sensitivity enables passive biocontainment wherein cellular fitness is unperturbed under standard laboratory conditions and markedly reduced in nature where fluoride is in sufficient abundance. This strategy is inherently robust as its efficacy relies on the absence of endogenous genes rather than the presence and activity of essential genes, which are subject to continuous selection pressure and neutral drift.

Biocontainment strain benchmarking. To further benchmark our strain, we assessed the strain with focus on four factors that would describe an ideal biocontainment strategy ${ }^{12}$ : (1) minimal fitness defects, (2) amenability to additional engineering, (3) escape rate below 1 in $10^{8}$ cells, and (4) genetic robustness. In contrast to stringent selection in the presence of fluoride, minimal fitness defects are desired in the absence of fluoride. Indeed, colony morphology is identical to that of the WT parent strain in the absence of fluoride (Fig. 2a). Similarly, the growth rate of the biocontainment strain $\left(\mu_{\max }=0.54 \mathrm{~h}^{-1}\right)$ is nearly identical to that of WT $\left(\mu_{\max }=0.61 \mathrm{~h}^{-1}\right)$ (Fig. 2c). Further, phenotypic homogeneity and mean fluorescence intensity (MFI) of the two strains are nearly identical upon expression of $y E G F P$ (Fig. $2 \mathrm{~d}$, e). The WT-level production of heterologous proteins is indicative of the biocontainment strain's capacity for additional engineering without pleiotropy. To determine the likelihood of biocontainment strain survival outside of controlled conditions, we determined the escape rate of our strain in the presence of $210.5 \mu \mathrm{M}$ and $5 \mathrm{mM} \mathrm{NaF}$. In both cases, the strain escape rate falls below the NIH guideline of 1 in $10^{8}$ cells (Supplementary Table 1). In the presence of $5 \mathrm{mM} \mathrm{NaF}$, the escape rate falls below the detection limit of our assay $\left(1\right.$ in $\left.10^{9}\right)$. While the observed escape rates reflect stringent growth inhibition in the presence of fluoride, these data do not necessarily reflect lethality. Relief of selection pressure could result in proliferation of the biocontainment strain. To assess whether fluoride is acting as a microbiostatic (i.e., growth inhibiting) or microbiocidal (i.e., lethal) agent, the biocontainment strain was grown in the presence of varying concentrations of fluoride, washed with sterile buffer, and used to inoculate fresh media. After $10 \mathrm{~h}$, cultures previously exposed to fluoride only reached $13-39 \%$ of the control culture concentration (Fig. 2f). The WT strain was also treated with $16 \mathrm{mM}$ fluoride prior to washing and resuspension in fresh media. Incubation of the WT strain with $16 \mathrm{mM}$ fluoride appears to affect the strain's growth, albeit to a lesser extent compared to the biocontainment strain. Thus, fluoride appears to act as a microbiostatic agent under the examined conditions and reduces, but not entirely, cell viability after treatment. While the fluoride treatment reported here may not serve as a direct substitute for conventional sterilization techniques (e.g., autoclaving), reduced fitness will impair the strain's capacity to persist in nature supporting the use of fluoride sensitivity as a biocontainment measure.

Concerning the fourth characteristic, genetic robustness, growth of the biocontainment strain is carried out in the absence of selection pressure, greatly reducing the likelihood of generating revertants or evolved fluoride resistance. This stands in contrast 


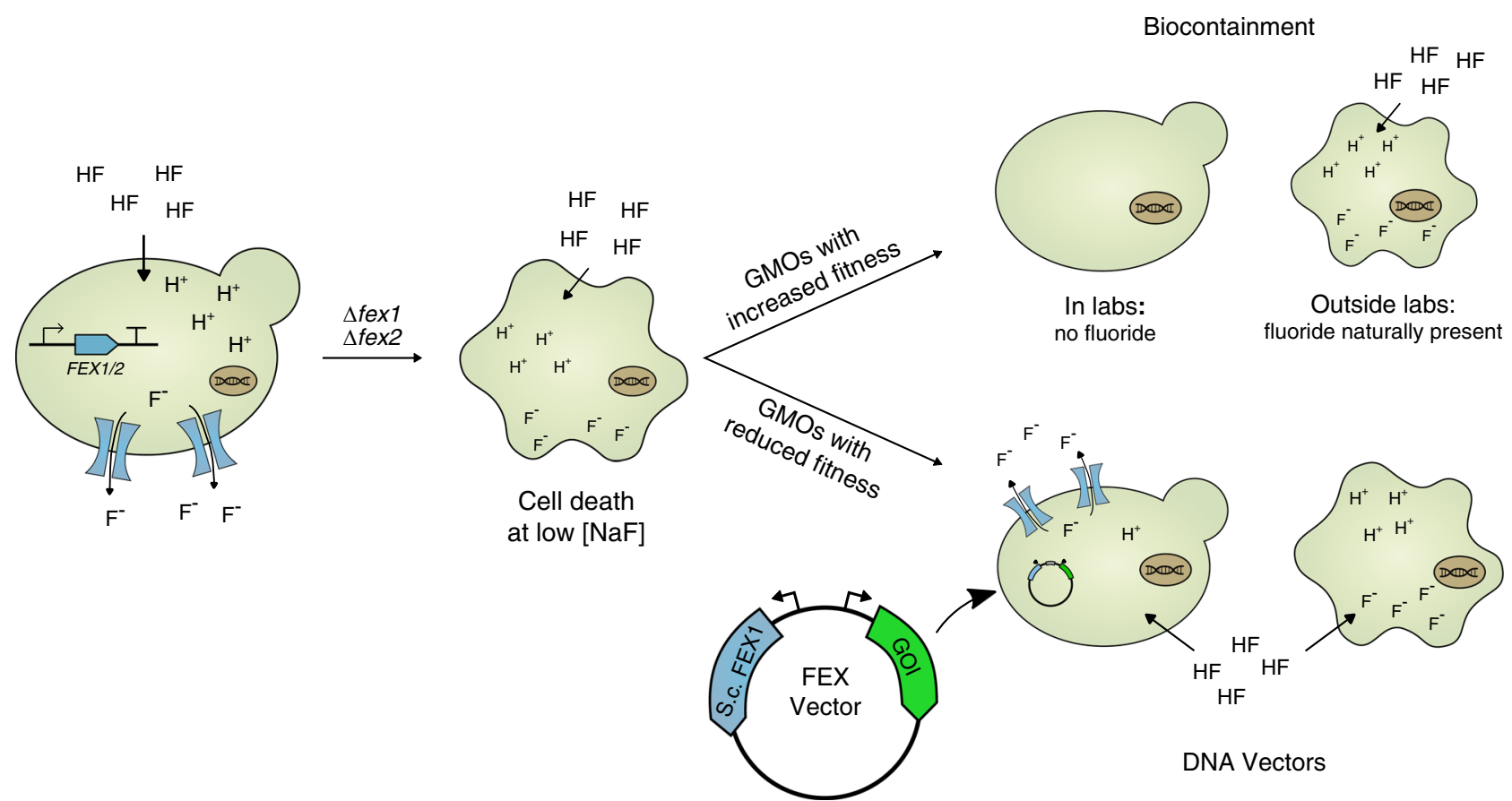

Fig. 1 Engineered fluoride sensitivity augments biocontainment and selection systems. Yeast cells lacking native fluoride exporter genes (FEX1/FEX2) are highly sensitized to low concentrations of fluoride yet retain wildtype phenotypes in the absence of the ion. Accordingly, this mechanism befits biocontainment of genetically modified organisms (GMOs) that pose ecological risks outside of laboratory environments. Alternatively, fluoride sensitivity can be leveraged to provide an alternative selection marker in GMOs with reduced fitness and likelihood of persistence in the environment.

to current yeast strategies ${ }^{16,17}$ in which cells are subject to continuous selection pressure to maintain functional components of the biocontainment system or circumvent the system altogether. In theory, our biocontainment system can be neutralized upon HGT of a functional FEX gene cassette from an organism in nature. While horizontal transfer of bacterial genes to $S$. cerevisiae may have occurred in nature ${ }^{27,28}$, a dedicated mechanism for free DNA uptake is yet to be discovered in $S$. cerevisiae ${ }^{28}$. Rather, bacterial, contact-dependent mechanisms (i.e., conjugation ${ }^{29}$ ) of DNA transfer are likely to have resulted in the presence of foreign genes in the $S$. cerevisiae genome. Accordingly, although possible, we posit that HGT of a FEX gene from a eukaryote is exceedingly unlikely to occur prior to death of the strain under selective pressure. Acquisition of fluoride tolerance could also be mediated through mating of the haploid biocontainment strain with a FEX-containing strain. However, a functional mating pathway is not necessary for cell viability, and yeasts are easily rendered incapable of mating through deletion of individual ste genes within the mating pathway $^{30}$.

Fluoride sensitivity enables fluoride-based vector selection. Complementary to a robust biocontainment system, cost-effective selection markers facilitate translation of lab-scale processes to the industrial scale. Maintenance of non-integrating plasmids or screening genomically integrated transformants using conventional selection agents are prominent operating costs at scale. In addition to elevating bioprocessing costs, the use of antibiotic selection introduces the risk of generating antibiotic-resistant microorganisms ${ }^{13}$. While auxotrophic selection eliminates the need for a selection agent, it necessitates the use of a defined medium, which can limit cellular growth and/or fitness. Further, in academic and research settings, there is a need for additional selection markers for genetic and metabolic engineering and synthetic biology. Exemplifying the need for alternative selection markers, Novo Nordisk developed the POT1 expression system, which enables production of insulin using $S$. cerevisiae in nutrient-rich media ${ }^{31,32}$. However, this system restricts the carbon source to glucose as the POT1 marker restores the glycolytic pathway in $S$. cerevisiae strains harboring a mutated copy of the native $t p i$. This growth scheme precludes use of galactoseinducible promoters, which are often used to limit deleterious effects of heterologous gene products such as membrane proteins. To address these issues, we constructed a set of DNA vectors containing fluoride selection markers. By replacing auxotrophic selection markers with the $S$. cerevisiae FEX1 gene in three commonly used vector backbones, we have constructed a set of yeast vectors that enable selective cell growth and production of heterologous proteins in rich, complex media containing low concentrations of $\mathrm{NaF}$. At recommended working concentrations, conventional antibiotics used with $S$. cerevisiae cost between US $\$ 32$ and $\$ 2175 / \mathrm{L}$, while $\mathrm{NaF}$ costs US\$0.04/L representing 3-5 orders of magnitude in potential savings (Supplementary Table 2) that become more prominent at industrial scales.

Characterization of FEX vectors. Introduction of a FEX vector into the knockout strain completely restores fluoride tolerance to WT levels (Fig. 3a). To challenge the robustness of the FEX vectors, we used the systems to express a human $G$ proteincoupled receptor, the adenosine $A_{2}$ a receptor $\left(A_{2} a R\right)$, as membrane protein expression often imparts metabolic burden by taxing the cell secretory pathway. Thus, we constructed integrating (pIFEX), centromeric (pCFEX1), and episomal (pEFEX1) vectors harboring $A_{2} a R-G F P$ and FEX cassettes (Fig. $3 b$ ). In these vectors, constitutive expression of the FEX gene is driven by the Ashbya gossypii TEF1 promoter, which is commonly used to express auxotrophic markers in yeast plasmids. Upon expression of $A_{2} a R-G F P$ from these plasmids, two distinct phenotypes emerge (Fig. 3c). Between $34 \%$ and $41 \%$ of cells harboring the non-integrating pCFEX1 or pEFEX1 constructs exhibit 
a

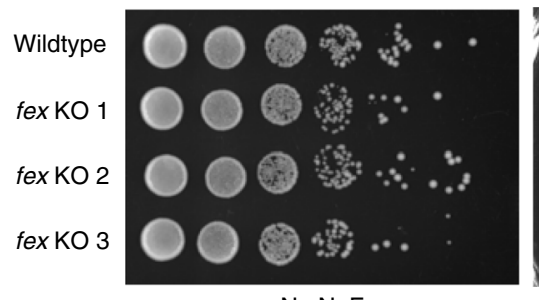

No NaF

b

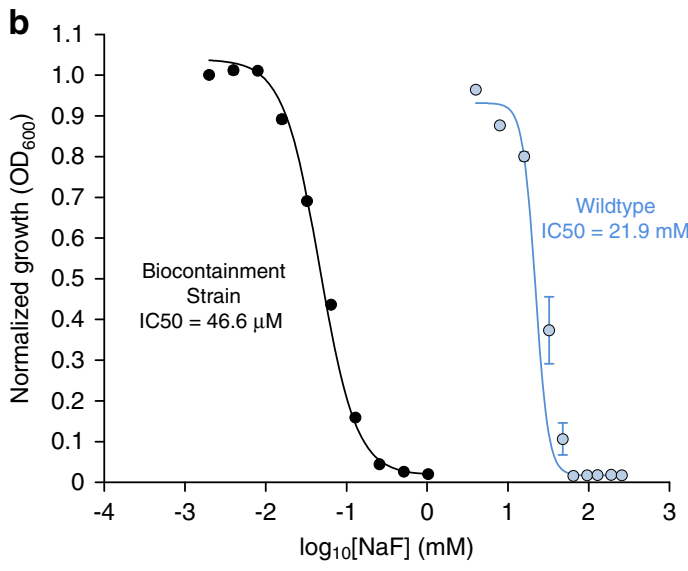

d

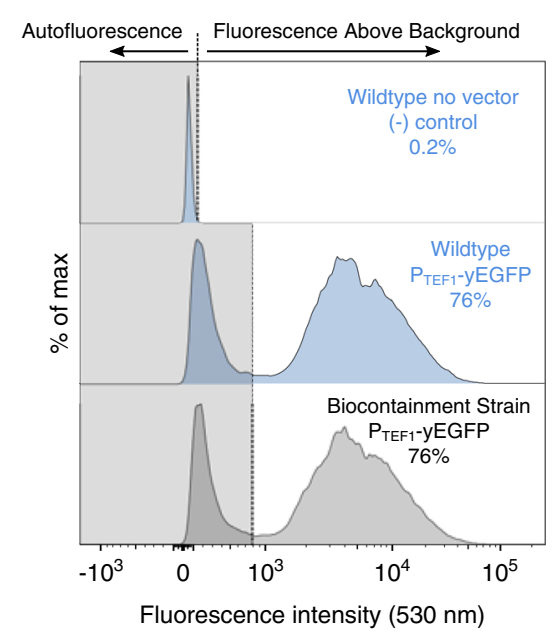

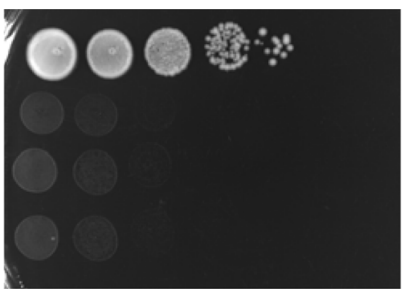

$210.5 \mu \mathrm{M} \mathrm{NaF}$

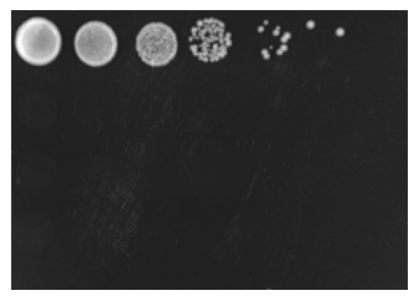

$5 \mathrm{mM} \mathrm{NaF}$

c
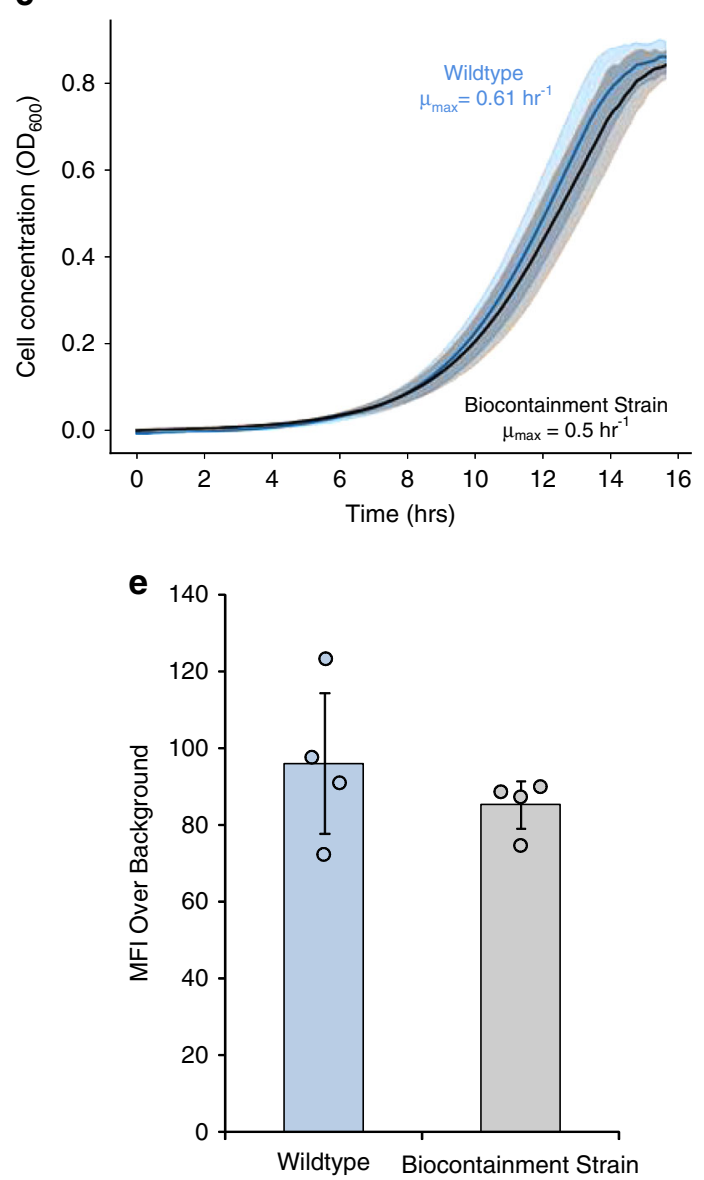

f
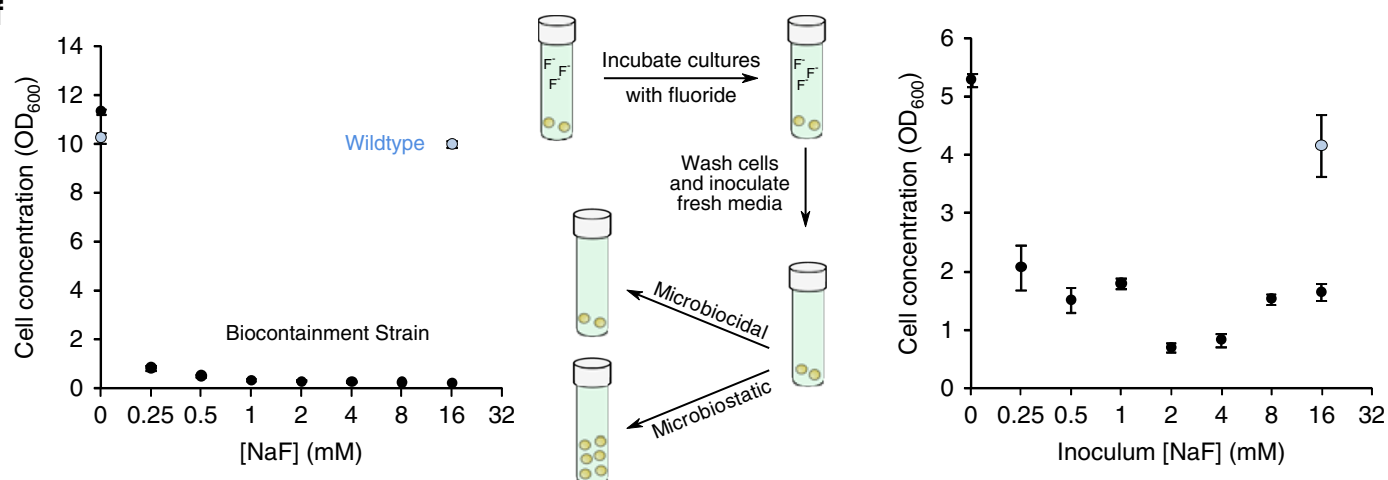

fluorescence intensities above autofluorescence. In contrast, 97\% of cells harboring the genomically integrated pIFEX construct displays fluorescence above background. The FEX vectors display varying degrees of similarity to their auxotrophic counterparts characterized previously ${ }^{33}$. Expression of $A_{2} a R-G F P$ from the high-copy backbones, pEFEX1 and pYES, yields similarly low fluorescence intensities, and the majority of each population displays fluorescence intensities comparable to autofluorescence. In contrast, $A_{2} a R-G F P$ expression from the auxotrophic low-copy vector, pYC, results in significantly greater MFI over background compared to the analogous FEX vector. Additionally, cells harboring pYC $\mathrm{A}_{2}$ aR-GFP exhibit a bimodal fluorescence 
Fig. 2 Yeast strains lacking FEX genes provide a stringent, passive biocontainment mechanism. a A yeast spotting assay demonstrates sensitivity of yeast lacking FEX genes to $\mu \mathrm{M}$ concentrations of $\mathrm{NaF}$. b Dose response curve of the biocontainment strain illustrates three orders of magnitude greater sensitivity to fluoride compared to the parent wildtype strain. $\mathbf{c}$ In the absence of fluoride, growth of the biocontainment and wildtype parent strains in YPD is nearly indistinguishable. $\mathbf{d}$, e Phenotypes of yEGFP production in the biocontainment and wildtype strains are also nearly identical, demonstrating the versatility of the biocontainment strain for engineering without deleterious effects associated with $F E X$ knockouts. $\mathbf{f}$ Cell growth is severely inhibited both in the presence of $\mathrm{NaF}$ as well as after washing and subculturing into fresh media, albeit to a lesser extent. In $\mathbf{b}, \mathbf{c}$, e, and $\mathbf{f}$, data represent the mean of three biological replicates, and error bars represent their standard deviation. The histograms presented in $\mathbf{d}$ correspond to representative samples. Source data are provided as a Source Data file.

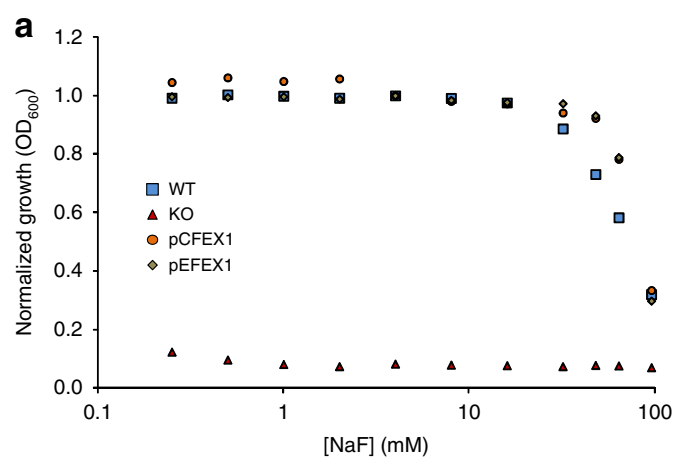

C

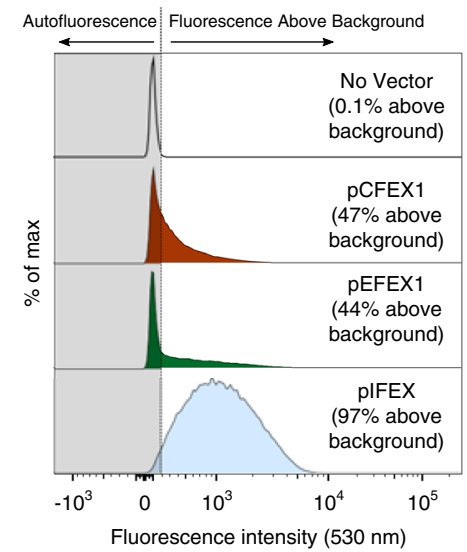

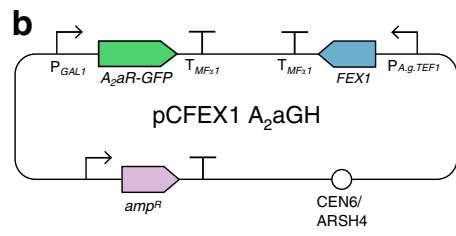
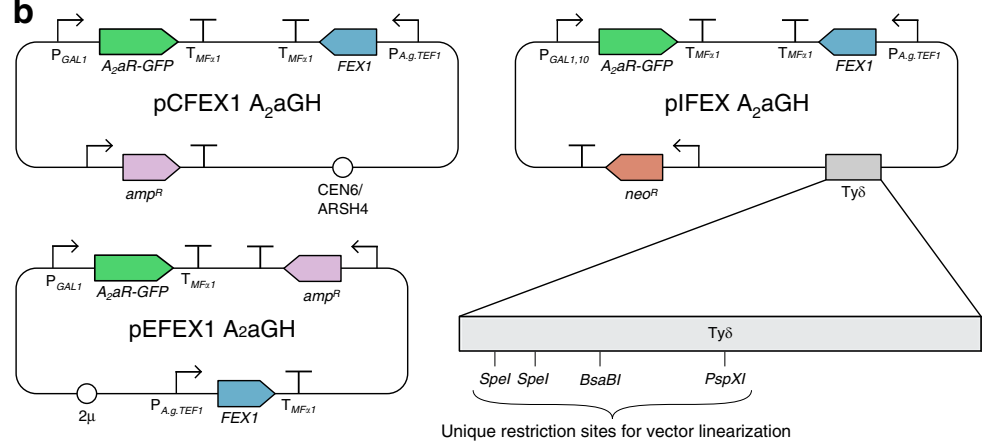

d
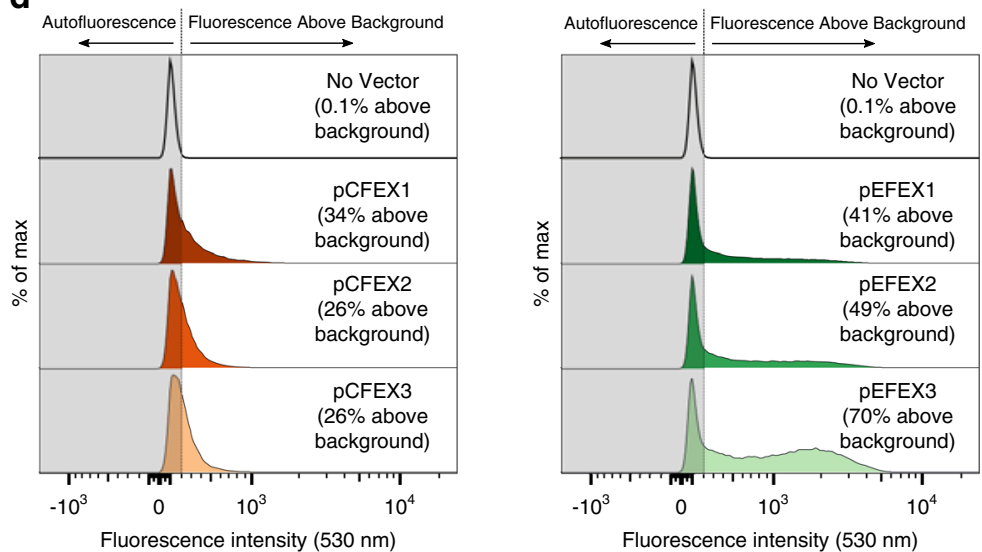

e

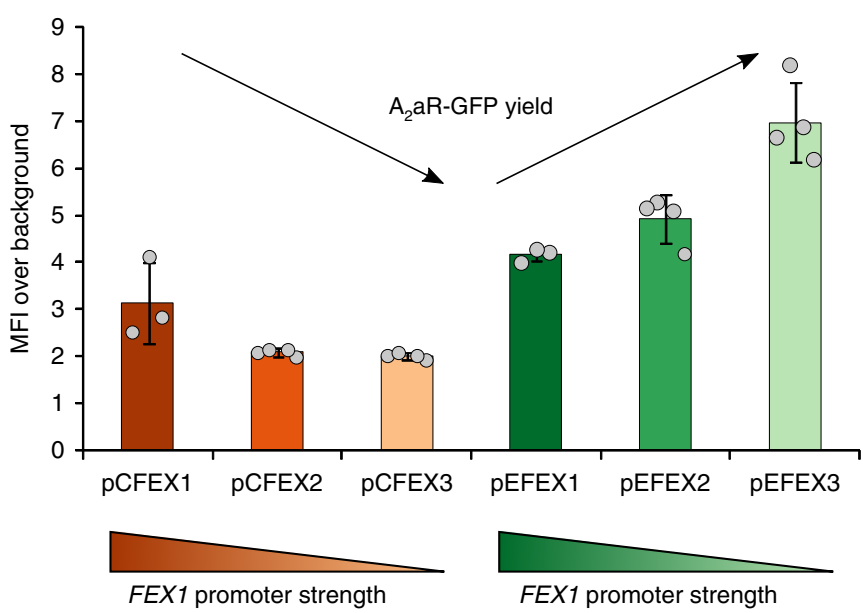

Fig. 3 Fluoride-sensitive yeast strains enable use of FEX as a selection marker. a While growth of the FEX knockout (KO) strain is inhibited by $\mu M$ concentrations of fluoride, transformation with a FEX-based vector restores fluoride resistance to wildtype levels. b Diagrams of centromeric ( $p C F E X 1$ ), episomal (pEFEX1), and integrating (pIFEX) vectors. c FACS analysis of $A_{2} a R-G F P$ expression from pCFEX1 and pEFEX1 reveals that a significant portion of cells exhibit background fluorescence intensities. In contrast, expression from the integrating pIFEX backbone yields a unimodal fluorescence distribution where $97 \%$ of the population displays fluorescence above background. d, e Replacement of the promoter driving FEX1 expression significantly influences $\mathrm{A}_{2} \mathrm{aR}$-GFP yield and phenotypic homogeneity in pEFEX but has less pronounced effects on pCFEX. In $\mathbf{c}$ and $\mathbf{d}$, histograms correspond to representative samples. Data present in a represents a single replicate for each strain. Data presented in e represents the mean of three biological replicates, and error bars represent their standard deviation. Source data are provided as a Source Data file. 
distribution, whereas a unimodal distribution is associated with pCFEX1 $\mathrm{A}_{2}$ aR-GFP where the majority of cells exhibit fluorescence intensities indistinguishable from autofluorescence. In all, $A_{2} a R$-GFP expression from the FEX vectors was initially poorer than expression from their auxotrophic counterparts.

Optimization of expression from FEX vectors. We sought to improve $\mathrm{A}_{2} \mathrm{aR}-\mathrm{GFP}$ yields associated with the non-integrating vectors, and initially hypothesized that plasmid loss contributes to the large fraction of cells displaying basal fluorescence intensity as observed previously ${ }^{33}$. Non-integrating plasmid loss is often a result of low mitotic stability. In other words, as a cell undergoes mitotic cell division, a non-integrating plasmid is more likely to be lost from the cell than one integrated into the genome. As demonstrated using the partially impaired LEU2-d and URA2-d selection markers, plasmid copy number can be modulated to cope with an imposed selection pressure ${ }^{34,35}$. Instead of modifying the FEX marker, we sought to impose stronger selection pressure by increasing the exogenous fluoride concentration. Fluoride transport across cellular membranes occurs primarily through diffusion of hydrogen fluoride $(\mathrm{HF})^{36}$; therefore, we generated a simple model of HF transport across the cell membrane, which indicates that a decrease of $1 \mathrm{pH}$ unit at $10 \mathrm{mM} \mathrm{NaF}$ produces a tenfold increase in fluoride flux (Supplementary Fig. 1). However, marginal differences in protein yield and phenotypic homogeneity are observed upon gene expression at lower pH (Supplementary Fig. 2).

Next, we hypothesized that the metabolic burden associated with producing two membrane proteins results in plasmid loss due to stress placed on the secretory pathway. This hypothesis is supported by the phenotype associated with the mitotically stable, integrating pIFEX vector. Therefore, we replaced the promoters driving FEX expression in pCFEX1 and pEFEX1 with weaker constitutive promoters (Supplementary Fig. 3). Upon replacing the A. gossypii TEF1 promoter in pEFEX1 with the weaker PGI1 (ref. ${ }^{37}$ ) (pEFEX2) and REV1 (ref. ${ }^{38}$ ) (pEFEX3) promoters, the proportion of cells exhibiting fluorescence intensity above background increased to $49 \%$ and $70 \%$, respectively (Fig. 3d). Total $\mathrm{A}_{2}$ aR-GFP yield also increases appreciably upon gene expression in pEFEX2 and pEFEX3 (Fig. 3e). Notably, expression of $A_{2} a R$-GFP from pEFEX3 yields a distinct subpopulation of cells exhibiting high fluorescence intensity resulting in a bimodal distribution (Fig. 3d). This phenotype represents a marked improvement compared to that observed using the auxotrophic high-copy vector described previously ${ }^{33}$. In contrast, promoter swapping in the pCFEX1 backbone did not yield appreciable differences in expression patterns (Fig. 3d) and even led to a reduction in $\mathrm{A}_{2} \mathrm{aR}-\mathrm{GFP}$ yield (Fig. $3 \mathrm{e}$ ). We speculate that the differences between the pCFEX and pEFEX systems arise due to differences in vector copy numbers. As pEFEX is likely maintained in higher copies, reduced FEX1 expression effectively reduces metabolic burden, which is common to secreted and membrane protein production from high-copy vectors ${ }^{33,39,40}$. In contrast, $\mathrm{A}_{2} \mathrm{aR}-\mathrm{GFP}$ production from the low-copy pCFEX vectors may not sufficiently tax the yeast secretory pathway to gain benefit from reduced FEX1 expression. Instead, lower FEX1 yields may increase sensitivity to exogenous fluoride leading to increased cell death.

\section{Discussion}

This work leverages fluoride sensitivity in biocontainment and plasmid systems. Our biocontainment strain offers several advantages over existing strategies. First, our strain is immediately amenable to academic and industrial applications as growth media is prepared using ultrapure water lacking fluoride.
Although residual fluoride is present in certain reagents, $\sim 7 \mu \mathrm{M}$ in YPD media ${ }^{22}$, the biocontainment strain showed no fitness defects compared to a WT control. Second, the knockouts can be easily introduced into any $S$. cerevisiae strain using accessible genetic engineering techniques such as CRISPR or Delitto Perfetto $^{41}$. These gene deletions can also be made directly in a previously modified organism to multiplex safeguards or introduce a new safeguard into an existing GMO. Third, in areas where fluoride is naturally abundant in the environment ${ }^{42}$, escapees are subject to lethal conditions without manual intervention. Despite the ubiquity of fluoride in the environment, laboratory conditions are stringently maintained. Thus, environmental fluoride is unlikely to contaminate bioreactors. Finally, other eukaryotic organisms containing fluoride transporter gene deletions exhibit sensitivity to fluoride ${ }^{22}$; thus, it is likely that our biocontainment strategy can be extended to a wide range of organisms relevant to biotechnology. The potential contamination of cultures by WT yeast represents a disadvantage of the FEX vector system, which is shared by auxotrophic selection strategies. While best laboratory practices will reduce the likelihood of contamination, FEX vectors can be used in combination with auxotrophy to minimize contaminations risks at the cost of precluding use of nutrient-rich, complex media.

In areas where fluoride is naturally abundant, the biocontainment platform is useful to reduce the fitness of GMOs that would otherwise prosper upon release into the environment; however, most genetic modifications will reduce an organism's ability to outcompete native microbes. Thus, we constructed a set of vectors that complements the $\Delta f e x$ background conferring fluoride tolerance to maintain heterologous DNA. While engineered fluoride sensitivity was previously used as the basis of a selection marker in a CRISPR/Cas system in Schizosaccharomyces pombe ${ }^{43}$, we extend the use of fluoride sensitivity to enable heterologous expression of any gene in the various DNA backbones available to the model yeast, $S$. cerevisiae. In contrast to auxotrophic markers, the FEX vectors enable use of rich media, which will not limit the growth or metabolism of engineered strains. Fluoride-based selection drastically reduces the cost of selection compared to antibiotics and precludes risks of generating antibiotic resistance. We expect that our contributions will be of immediate use in both academic and industrial settings to advance efforts in synthetic biology.

\section{Methods}

Plasmid construction. In non-integrating yeast vectors, auxotrophic markers are often placed under the control of the A. gossypii $\mathrm{P}_{\mathrm{TEF} 1}$ promoter. Thus, we first constructed an A. gossypii $\mathrm{P}_{\mathrm{TEF} 1}-\mathrm{S}$. cerevisiae FEX1- $\mathrm{T}_{\mathrm{MF} \alpha 1}$ cassette in the pITy backbone through two steps. First, primers 1 and 2 were used to append EcoRI and EagI sites to an A. gossypii $\mathrm{P}_{\mathrm{TEF} 1}$ fragment amplified from pSVA13 (Supplementary Table 3). This fragment and pITy $\mathrm{A}_{2}$ aGH were digested with EcoRI-HF and EagI$H F$ and ligated to generate an intermediate pITy A. gossypii $\mathrm{P}_{\mathrm{TEF} 1} \mathrm{~A}_{2} \mathrm{aGH}$ construct. Second, primers 3 and 4 were used to amplify S. cerevisiae FEX1 from BJ5465 gDNA extracted using the protocol provided by Lõoke et al. ${ }^{44}$. This fragment and pITy A. gossypii $\mathrm{P}_{\mathrm{TEF} 1} \mathrm{~A}_{2} \mathrm{aGH}$ were digested with EagI-HF and AflII and ligated to generate pITy A. gossypii $\mathrm{P}_{\mathrm{TEF1}}-\mathrm{S}$. cerevisiae FEX1- $\mathrm{T}_{\mathrm{MF} \alpha 1}$. $\mathrm{USER}^{45}$ cloning was used to subclone the A. gossypii $\mathrm{P}_{\mathrm{TEF1}}-\mathrm{S}$. cerevisiae FEX1- $\mathrm{T}_{\mathrm{MFa} 1}$ cassette, which was amplified using primers 5 and 6 , into the pYC2/CT and pYES2 backbones amplified using primer pairs 7 and 8 and 8 and 9 , respectively. The resulting constructs were named pCFEX1 $\mathrm{A}_{2} \mathrm{aGH}$ and pEFEX1 $\mathrm{A}_{2} \mathrm{aGH}$, respectively. The A. gossypii $\mathrm{P}_{\mathrm{TEF} 1}$ promoters were swapped with $\mathrm{P}_{\mathrm{PGI1}}$ and $\mathrm{P}_{\mathrm{REV} 1}$ promoters amplified from BJ5465 gDNA using primer pairs 10 and 11 and 12 and 13, respectively. pCFEX1 $\mathrm{A}_{2} \mathrm{aGH}$ and pEFEX1 $\mathrm{A}_{2} \mathrm{aGH}$ backbones were amplified using primer pairs 7 and 14 and 9 and 14, respectively, to mediate promoter swapping through USER cloning. The pCFEX2 and pEFEX2 backbones carry the $\mathrm{P}_{\text {PGI1 }}$ promoter, and the pCFEX 3 and pEFEX 3 backbones carry the $\mathrm{P}_{\mathrm{REV} 1}$ promoter. The integrating pIFEX $\mathrm{A}_{2} \mathrm{aGH}$ construct was generated through USER cloning after amplifying pITy $\mathrm{A}_{2} \mathrm{aGH}$ using primers 15 and 16 and the A. gossypii $\mathrm{P}_{\mathrm{TEF}}-\mathrm{S}$. cerevisiae FEX1- $\mathrm{T}_{\mathrm{MF} \alpha 1}$ cassette using primers 5 and 6. The pIFEX $\mathrm{A}_{2} \mathrm{aGH}$ construct was designed to retain the NEO CDS to facilitate cloning in Escherichia coli using kanamycin; thus, the vector also confers G418 resistance to yeast. All constructs 
were sequence-verified using Sanger sequencing (Genewiz) and transformed into $S$. cerevisiae using the high-efficiency lithium acetate protocol ${ }^{46}$. We found that a recovery period is necessary to obtain yeast transformants using the FEX vectors. Following resuspension in YPD, transformed yeast cells were incubated at $30^{\circ} \mathrm{C}$ for at least $3 \mathrm{~h}$ prior to plating on YPD plates containing $210.5 \mu \mathrm{M} \mathrm{NaF}$. Yeast transformed with pIFEX $\mathrm{A}_{2} \mathrm{aGH}$ were plated on YPD supplemented with $10 \mathrm{mM}$ $\mathrm{NaF}$ to promote increased gene dosage. The $\mathrm{pRS} 315 \mathrm{P}_{\mathrm{TEF1}}-y E G F P$ construct was generated from pRS315 $\mathrm{P}_{T E F 1}-\mathrm{yEGFP}-\mathrm{Cln} 2$, which was cloned for a separate study, in a series of steps. First, the pRS315 backbone was digested with EagI, blunted with Klenow fragment, and digested with SpeI. The $\mathrm{P}_{T E F 1}$-yEGFP-Cln2 cassette was amplified from YEp351 $\mathrm{P}_{T E F 1}-\mathrm{yEGFP-Cln} 2$ using universal M13 forward and reverse primers, then the amplicon was digested with SpeI to mediate directional cloning into pRS315. The backbone and insert were ligated and transformed into $E$. coli. Next, USER cloning mediated construction of $\mathrm{pRS}_{15} \mathrm{P}_{\text {TEF } 1}-\mathrm{yEGFP}$ from the Cln2-tagged plasmids. Primers 17 and 18 were used to amplify the plasmid excluding the $\mathrm{Cln} 2$ tag and introducing two stop codons at the $3^{\prime}$-end of the $y E G F P$ coding sequence.

Yeast strains and culturing conditions. S. cerevisiae strain BJ5465 (Mata ura3-52

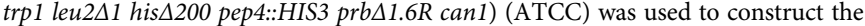
biocontainment strain BJ5465 fex1::GSHU $\triangle$ fex2 using the Delitto Perfetto method ${ }^{47}$. Primers 19 and 20 were used to integrate the GSHU cassette at the FEXI locus, and primers 19 and 21 were used to integrate the CORE-Kp53 cassette into the FEX2 locus. Subsequently, primers 22 and 23 were used to remove the COREKp53 cassette. Culture maintenance and gene expression were carried out using YPD medium at $30^{\circ} \mathrm{C}$ with shaking at 225 r.p.m. Cultures harboring pCFEX and pEFEX plasmids were maintained in YPD supplemented with $2 \mathrm{mM} \mathrm{NaF}$.

Fluorescence-activated cell sorting. Yeast cultures were diluted to an $\mathrm{OD}_{600}=$ 1.0 in $1 \times$ phosphate buffered saline (PBS) prior to all fluorescence-activated cell sorting (FACS) analyses. Approximately 60,000 cells were analyzed from each sample using a $488-\mathrm{nm}$ laser and $530 / 30 \mathrm{~nm}$ bandpass filter using the gating strategy illustrated in Supplementary Fig. 4. All analyses were conducted using a BD FACSAria I flow cytometer and FlowJo v10. To analyze $y E G F P$ expression, WT and biocontainment strains harboring $\mathrm{pRS} 315 \mathrm{P}_{\mathrm{TEF} 1}-y E G F P$ were used to inoculate $5 \mathrm{~mL}$ synthetic dextrose medium supplemented with amino acids lacking leucine $(\mathrm{SD}-\mathrm{leu})^{47}$. Following overnight growth, cells were resuspended in $1 \times$ PBS and analyzed using FACS as described above. To analyze $A_{2} a R$-GFP expression from pCFEX and pEFEX vector backbones, knockout strains carrying the vectors were first cultured overnight in YPD medium supplemented with $2 \mathrm{mM} \mathrm{NaF}$ at $30^{\circ} \mathrm{C}$ with shaking at 225 r.p.m. Following overnight growth, each culture was subcultured into YP medium supplemented with $2 \%(\mathrm{w} / \mathrm{v})$ raffinose (YPR) and $2 \mathrm{mM}$ $\mathrm{NaF}$ at an initial $\mathrm{OD}_{600}$ of 0.5 . Following $\sim 10 \mathrm{~h}$ of shaking at $30^{\circ} \mathrm{C}$, each culture was subcultured into YP medium supplemented with $2 \%(\mathrm{w} / \mathrm{v})$ raffinose, $2 \%(\mathrm{w} / \mathrm{v})$ galactose (YPRG), and $2 \mathrm{mM} \mathrm{NaF}$ to induce $A_{2} a R$-GFP expression. Cultures were incubated with shaking at $30^{\circ} \mathrm{C}$ overnight prior to flow cytometric analysis. Analysis of $A_{2} a R-G F P$ expression from the pIFEX backbone was accomplished using a similar induction scheme in the absence of NaF. Knockout strains harboring integrated pIFEX $\mathrm{A}_{2} \mathrm{aR}$-GFP cassettes were cultured in YPD medium overnight at $30^{\circ} \mathrm{C}$ with shaking at 225 r.p.m. Subsequently, cultures were subcultured into YPR medium and incubated at $30^{\circ} \mathrm{C}$ with shaking. After $\sim 10 \mathrm{~h}$, $\mathrm{A}_{2} a R$-GFP expression was induced in each culture through subculturing into YPRG medium and incubation at $30^{\circ} \mathrm{C}$ with shaking overnight.

Dilution spotting. Yeast cultures were grown to an $\mathrm{OD}_{600} \sim 3$ prior to dilution to an $\mathrm{OD}_{600}=2.5$ in sterile YPD. Diluted cells were used to prepare serial dilutions up to $10^{-5}$ in tenfold increments. A total of $5 \mu \mathrm{L}$ of each dilution was spotted onto solid media using a multichannel pipette. Plates were allowed to dry at room temperature prior to overnight incubation at $30^{\circ} \mathrm{C}$.

Fluoride dose response assay. WT and biocontainment strain cultures were grown in biological triplicate overnight in YPD at $30^{\circ} \mathrm{C}$ with shaking at 225 r.p.m. In the morning, the cultures were used to inoculate $5 \mathrm{~mL}$ fresh YPD at an initial $\mathrm{OD}_{600}$ of 0.15 . Cultures were incubated with shaking at $30^{\circ} \mathrm{C}$ for $7 \mathrm{~h}$, reaching $\mathrm{OD}_{600}$ values near 2 , and used to inoculate $3 \mathrm{~mL}$ YPD in individual wells of a 24 well block (Qiagen \#19583) containing serially diluted concentrations of $\mathrm{NaF}$ and covered with a Breathe Easier sealing membrane (Sigma-Aldrich Z763624). Following overnight shaking at $30^{\circ} \mathrm{C}, \mathrm{OD}_{600}$ values were measured for cultures in each well.

Growth curves. To generate growth curves, the WT and biocontainment strains were used to inoculate $5 \mathrm{~mL}$ YPD cultures, which were grown overnight at $30^{\circ} \mathrm{C}$ with shaking at 225 r.p.m. Cultures were used to inoculate $1 \mathrm{~mL}$ YPD in individual wells of a 24-well plate (Corning 3526), at an initial $\mathrm{OD}_{600}=0.02$. Cell growth was monitored using a Tecan Spark microplate reader maintained at $30{ }^{\circ} \mathrm{C}$ with orbital shaking at 180 r.p.m. and $3 \mathrm{~mm}$ amplitude. $\mathrm{OD}_{600}$ measurements were taken every $10 \mathrm{~min}$ with $50 \mathrm{~ms}$ settling time prior to each reading. Specific growth rates were calculated by fitting data to the logistic function ${ }^{48}$ (Eq. (1)):

$$
N(t)=N_{0}+\frac{N_{\text {asymp }}-N_{0}}{1+e^{\left[k\left(t_{\mathrm{c}}-t\right)\right]}},
$$

where $N_{0}$ is the initial number of cells, $N_{\text {asymp }}$ is the maximal number of cells approached during stationary phase, $k$ is the growth rate, and $t_{\mathrm{c}}$ is the time at which the growth curve exhibits an inflection point.

Escape rate determination. To determine the escape rate, the biocontainment strain was grown overnight in biological triplicate in YPD at $30^{\circ} \mathrm{C}$ with shaking at 225 r.p.m. In the morning, $\sim 50$ colony forming units (CFUs) of each replicate culture were plated onto YPD media assuming a conversion factor of $10^{7} \mathrm{CFU} / \mathrm{mL} /$ $\mathrm{OD}_{600}$. Using the same conversion factor, $10^{8}$ and $10^{9} \mathrm{CFUs}$ of each replicate culture were plated onto YPD supplemented with $210.5 \mu \mathrm{M}$ and $5 \mathrm{mM}$ NaF. Following incubation of plates at $30^{\circ} \mathrm{C}$ for 2 days, CFUs were counted. The CFU values obtained for the YPD control plates were used to correct the CFU/mL/ $\mathrm{OD}_{600}$ conversion factor and to calculate the total number of cells plated on each plate.

pH Buffering experiment. Individual colonies were used to inoculate $5 \mathrm{~mL}$ YPD medium. Cultures were grown overnight at $30^{\circ} \mathrm{C}$ with shaking at 225 r.p.m. Following overnight growth, cells were used to inoculate $\mathrm{YPR}$ at an initial $\mathrm{OD}_{600}=0.2$. Cultures were grown for $7-8 \mathrm{~h}$ at $30^{\circ} \mathrm{C}$ with shaking at $225 \mathrm{r} . \mathrm{p} . \mathrm{m}$. and used to inoculate $5 \mathrm{~mL}$ YPRG supplemented with $10 \mathrm{mM} \mathrm{NaF}$ at an initial $\mathrm{OD}_{600}=0.2$. One set of cultures were used to inoculate $5 \mathrm{~mL}$ YPRG buffered to $\mathrm{pH}=6$ using $100 \mathrm{mM}$ MES (Sigma-Aldrich, St. Louis, MO, USA). Following overnight growth, cells were prepared for FACS analysis as described above.

Microbiostatic/microbiocidal assay. WT and biocontainment strain cultures were grown overnight and subcultured into $5 \mathrm{~mL}$ YPD at an initial $\mathrm{OD}_{600}$ of 0.1 . Cultures were incubated with shaking at $30^{\circ} \mathrm{C}$ for $\sim 10 \mathrm{~h}$ to an $\mathrm{OD}_{600}$ between 2 and 4 and used to inoculate $3 \mathrm{~mL}$ YPD in individual wells of a 24-well block containing serially diluted concentrations of $\mathrm{NaF}$ and covered with a Breathe Easier sealing membrane. Following overnight incubation with shaking at $30^{\circ} \mathrm{C}$, the $\mathrm{OD}_{600}$ values were measured for cultures in each well. The equivalent of $0.2 \mathrm{OD}$ $\mathrm{mL}$ of cells were spun at $3000 \times g$ for $30 \mathrm{~s}$, washed with sterile $1 \times \mathrm{PBS}$, and used to inoculate $3 \mathrm{~mL}$ fresh YPD in a sterile well of a 24-well block, which was covered with a Breathe Easier sealing membrane. Upon overnight growth, the $\mathrm{OD}_{600}$ values were measured for cultures in each well.

Modeling cellular fluoride uptake. Fluoride uptake was approximated by modeling the transport of HF across the cell membrane. First, the concentration of HF in bulk is calculated from the exogenous $\mathrm{NaF}$ concentration using Eq. (2):

$$
[\mathrm{HF}]=\frac{[\mathrm{NaF}]}{\left(\frac{10^{-3.17}}{10^{-p H}}\right)+1} .
$$

The above equation takes into account the pKa of $\mathrm{HF}$ at $25^{\circ} \mathrm{C}$, which is equal to 3.17. Next, the general transport equation (Eq. (3)) can be solved for the timedependent concentration of $\mathrm{NaF}$ inside of the cell:

$$
\frac{\partial C}{\partial t}=D \nabla^{2} C
$$

where $C$ is the time-dependent concentration of fluoride and $D$ is the diffusion coefficient of fluoride. Solving Eq. (3) for C:

$$
C_{\mathrm{i}}(t)=C_{0}\left(1-e^{-\frac{A P}{V} t}\right)
$$

where $C_{\mathrm{i}}(t)$ is the intracellular fluoride concentration, $C_{0}$ is the bulk fluoride concentration, $A$ is the membrane surface area, $P$ is the permeability constant, and $V$ is the cell volume. The permeability constant used for HF in the cell membrane is $0.0002 \mathrm{~cm} / \mathrm{s}$ as calculated by Gutknecht et al. ${ }^{49}$. The cell surface area and volume were estimated from figures provided on bionumbers.hms.harvard.edu.

Now, the fluoride concentrations can be used to solve for the flux, $J$, of fluoride across the cell:

$$
\begin{gathered}
J=-P\left(C_{\mathrm{i}}-C_{0}\right), \\
J=P C_{0} e^{-\frac{A P}{V} t} .
\end{gathered}
$$

Now, Eq. (6) gives the flux of fluoride across the cell membrane given a bulk concentration of fluoride, which is dictated by the exogenous $\mathrm{NaF}$ concentration as calculated in Eq. (2).

Reporting Summary. Further information on research design is available in the Nature Research Reporting Summary linked to this article.

\section{Data availability}

The data that support the findings of this study are available in this manuscript, the supplementary materials, and from the corresponding author upon request. The 
biocontainment strain, BJ5465 fex1::GSHU $\Delta$ fex2, will be made available via reasonable request to M.A.O. The pIFEX, pEFEX, and pCFEX vectors and associated vector maps described in this work are available from Addgene (deposit number 78647). Source data are provided with this paper.

Received: 13 June 2020; Accepted: 2 October 2020;

Published online: 29 October 2020

\section{References}

1. Hayden, E. C. Synthetic-biology firms shift focus. Nature 505, 598 (2014).

2. Li, M. \& Borodina, I. Application of synthetic biology for production of chemicals in yeast Saccharomyces cerevisiae. FEMS Yeast Res. 15, 1-12 (2015).

3. Collins, J. J. \& Khalil, A. S. Synthetic biology: applications come of age. Nat. Rev. Genet. 11, 367-379 (2010).

4. Jensen, M. K. \& Keasling, J. D. Recent applications of synthetic biology tools for yeast metabolic engineering. FEMS Yeast Res. 15, 1-10 (2014).

5. Gibson, D. G. et al. Complete chemical synthesis, assembly, and cloning of a Mycoplasma genitalium genome. Science 319, 1215-1220 (2008).

6. Gibson, D. G. et al. Creation of a bacterial cell controlled by a chemically synthesized genome. Science 52, 52-56 (2012).

7. Hutchison, C. A. et al. Design and synthesis of a minimal bacterial genome. Science 351, aad6253 (2016).

8. Ostrov, N. et al. Design, synthesis, and testing toward a 57-codon genome. Science 353, 819-822 (2016).

9. Richardson, S. M. et al. Design of a synthetic yeast genome. Science 355, 1040-1044 (2017).

10. Berg, P., Baltimore, D., Brenner, S., Roblin, R. O. \& Singer, M. F. Summary statement of the Asilomar conference on recombinant DNA molecules. Proc. Natl Acad. Sci. USA 72, 1981-1984 (1975).

11. Moe-Behrens, G. H. G., Davis, R. \& Haynes, K. A. Preparing synthetic biology for the world. Front. Microbiol. 4, 44-49 (2013).

12. Lee, J. W., Chan, C. T. Y., Slomovic, S. \& Collins, J. J. Next-generation biocontainment systems for engineered organisms. Nat. Chem. Biol. 14, 1 (2018).

13. O'Neill, J. Tackling drug-resistant infections globally: final report and recommendations. https://amr-review.org/sites/default/files/160518_Final\% 20paper with\%20cover.pdf (2016).

14. Mignon, C., Sodoyer, R. \& Werle, B. Antibiotic-free selection in biotherapeutics: now and forever. Pathogens 4, 157-181 (2015).

15. Wright, O., Stan, G.-B. \& Ellis, T. Building-in biosafety for synthetic biology. Microbiology 159, 1221-1235 (2013)

16. Cai, Y. et al. Intrinsic biocontainment: Multiplex genome safeguards combine transcriptional and recombinational control of essential yeast genes. Proc. Natl Acad. Sci. USA 112, 1803-1808 (2015).

17. Agmon, N. et al. Low escape-rate genome safeguards with minimal molecular perturbation of Saccharomyces cerevisiae. Proc. Natl Acad. Sci. USA 114, E1470-E1479 (2017)

18. Redden, H., Morse, N. \& Alper, H. S. The synthetic biology toolbox for tuning gene expression in yeast. FEMS Yeast Res. 15, 1-10 (2014).

19. Da Silva, N. A. \& Srikrishnan, S. Introduction and expression of genes for metabolic engineering applications in Saccharomyces cerevisiae. FEMS Yeast Res. 12, 197-214 (2012).

20. Wang, G., Huang, M. \& Nielsen, J. Exploring the potential of Saccharomyces cerevisiae for biopharmaceutical protein production. Curr. Opin. Biotechnol. 48, 77-84 (2017).

21. Borodina, I. \& Nielsen, J. Advances in metabolic engineering of yeast Saccharomyces cerevisiae for production of chemicals. Biotechnol. J. 9, 609-620 (2014).

22. Li, S. et al. Eukaryotic resistance to fluoride toxicity mediated by a widespread family of fluoride export proteins. Proc. Natl Acad. Sci. USA 110, 19018-19023 (2013).

23. Seppälä, S., Yoo, J. I., Yur, D. \& O’Malley, M. A. Heterologous transporters from anaerobic fungi bolster fluoride tolerance in Saccharomyces cerevisiae. Metab. Eng. Commun. 9, e00091 (2019).

24. EPA. National Primary Drinking Water Regulations. https://www.epa.gov/ ground-water-and-drinking-water/national-primary-drinking-waterregulations (2020).

25. McMahon, P. B., Brown, C. J., Johnson, T. D., Belitz, K. \& Lindsey, B. D. Fluoride occurrence in United States groundwater. Sci. Total Environ. 732, 139217 (2020)

26. Edmunds, W. M. \& Smedley, P. L. Fluoride in natural water. In Essentials of Medical Geology: Revised Edition (eds Selinus, O. et al.) 311-336 (Springer, 2013).
27. Gojković, Z. et al. Horizontal gene transfer promoted evolution of the ability to propagate under anaerobic conditions in yeasts. Mol. Genet. Genomics 271, 387-393 (2004).

28. Hall, C., Brachat, S. \& Dietrich, F. S. Contribution of horizontal gene transfer to the evolution of Saccharomyces cerevisiae. Eukaryot. Cell 4, 1102-1115 (2005).

29. Heinemann, J. A. \& Sprague, G. F. Bacterial conjugative plasmids mobilize DNA transfer between bacteria and yeast. Nature 340, 205-209 (1989).

30. Hartwell, L. H. Mutants of Saccharomyces cerevisiae unresponsive to cell division control by polypeptide mating hormone. J. Cell Biol. 85, 811-822 (1980).

31. Kawasaki, G. H. \& Bell, L. Stable DNA Constructs. US Patent 5871957 (1999).

32. Liu, Z., Tyo, K. E. J., Martínez, J. L., Petranovic, D. \& Nielsen, J. Different expression systems for production of recombinant proteins in Saccharomyces cerevisiae. Biotechnol. Bioeng. 109, 1259-1268 (2012).

33. Yoo, J. I. \& O’Malley, M. A. Tuning vector stability and integration frequency elevates functional GPCR production and homogeneity in Saccharomyces cerevisiae. ACS Synth. Biol. 7, 1763-1772 (2018).

34. Erhart, E. \& Hollenberg, C. P. The presence of a defective LEU2 gene on $2 \mu$ DNA recombinant plasmids of Saccharomyces cerevisiae is responsible for curing and high copy number. J. Bacteriol. 156, 625-635 (1983).

35. Loison, G. et al. High level of expression of a protective antigen of schistosomes in Saccharomyces cerevisiae. Yeast 5, 497-507 (1989).

36. Barbier, O., Arreola-Mendoza, L. \& Del Razo, L. M. Molecular mechanisms of fluoride toxicity. Chem. Biol. Interact. 188, 319-333 (2010).

37. Sun, J. et al. Cloning and characterization of a panel of constitutive promoters for applications in pathway engineering in Saccharomyces cerevisiae. Biotechnol. Bioeng. 109, 2082-2092 (2012).

38. Lee, M. E., DeLoache, W. C., Cervantes, B. \& Dueber, J. E. A highly characterized yeast toolkit for modular, multipart assembly. ACS Synth. Biol. 4, 975-986 (2015)

39. Wittrup, K. D., Robinson, A. S., Parekh, R. N. \& Forrester, K. J. Existence of an optimum expression level for secretion of foreign proteins in yeast. Ann. N. Y. Acad. Sci. 745, 321-330 (1994).

40. Parekh, R., Forrester, K. \& Wittrup, D. Multicopy overexpression of bovine pancreatic trypsin inhibitor saturates the protein folding and secretory capacity of Saccharomyces cerevisiae. Protein Expr. Purif. 6, 537-545 (1995)

41. Storici, F. \& Resnick, M. A. The delitto perfetto approach to in vivo sitedirected mutagenesis and chromosome rearrangements with synthetic oligonucleotides in yeast. Methods Enzymol. 409, 329-345 (2006).

42. Brindha, K. \& Elango, L. Fluoride in Groundwater: Causes, Implications and Mitigation Measures. In Fluoride: Properties, Applications, and Environmental Management. (ed Monroy, S. D.) 111-136 (Nova Publishers, 2011).

43. Fernandez, R. \& Berro, J. Use of a fluoride channel as a new selection marker for fission yeast plasmids and application to fast genome editing with CRISPR/ Cas9. Yeast 33, 549-557 (2016).

44. Lõoke, M., Kristjuhan, K. \& Kristjuhan, A. Extraction of genomic DNA from yeasts for PCR-based applications. Biotechniques 50, 325-328 (2011).

45. Nørholm, M. H. H. A mutant Pfu DNA polymerase designed for advanced uracil-excision DNA engineering. BMC Biotechnol. 10, 21 (2010).

46. Gietz, R. D. \& Woods, R. A. Transformation of yeast by lithium acetate/singlestranded carrier DNA/polyethylene glycol method. Methods Enzymol. 350, 87-96 (2002).

47. Wittrup, K. D. \& Benig, V. Optimization of amino acid supplements for heterologous protein secretion in Saccharomyces cerevisiae. Biotechnol. Tech. 8, 161-166 (1994).

48. Micha, P. \& Corradini, M. G. Microbial growth curves: what the models tell us and what they cannot. Crit. Rev. Food Sci. Nutr. 51, 917-945 (2011)

49. Gutknecht, J. \& Walter, A. Hydrofluoric and nitric acid transport through lipid bilayer membranes. Biochim. Biophys. Acta - Biomambr. 644, 153-156 (1981).

\section{Acknowledgements}

We thank Dr. Stephen Streatfield for helpful discussions regarding biocontainment systems and Professor Simon Avery for pSVA13. J.I.Y. acknowledges support from a National Science Foundation Graduate Research Fellowship under grant no. 1650114. The authors further acknowledge funding support from the National Science Foundation (MCB-1553721).

\section{Author contributions}

J.I.Y. and M.A.O. conceived the study, designed the experiments, analyzed the data, and wrote the manuscript. J.I.Y. and S.S. conducted the experiments.

\section{Competing interests}

The authors declare the following competing interests: J.I.Y. and M.A.O. are authors on a patent application, application number 63072933, which has been filed. All other authors declare no competing interests. 


\section{Additional information}

Supplementary information is available for this paper at https://doi.org/10.1038/s41467020-19271-1.

Correspondence and requests for materials should be addressed to M.A.O.

Peer review information Nature Communications thanks Michael Smanski and the other, anonymous, reviewer(s) for their contribution to the peer review of this work. Peer reviewer reports are available.

Reprints and permission information is available at http://www.nature.com/reprints

Publisher's note Springer Nature remains neutral with regard to jurisdictional claims in published maps and institutional affiliations. (c) (i) Open Access This article is licensed under a Creative Commons Attribution 4.0 International License, which permits use, sharing, adaptation, distribution and reproduction in any medium or format, as long as you give appropriate credit to the original author(s) and the source, provide a link to the Creative Commons license, and indicate if changes were made. The images or other third party material in this article are included in the article's Creative Commons license, unless indicated otherwise in a credit line to the material. If material is not included in the article's Creative Commons license and your intended use is not permitted by statutory regulation or exceeds the permitted use, you will need to obtain permission directly from the copyright holder. To view a copy of this license, visit http://creativecommons.org/ licenses/by/4.0/.

(C) The Author(s) 2020 